$$
\text { ANL/UPD/CP - } 102591
$$

High-resolution atomic distribution functions of disordered materials by high-energy $x$-ray diffraction

V. Petkov, S.J.L. Billinge, S. D. Shastri and B. Himmel

User Program Division

Argonne National Laboratory

Argonne, IL 60439

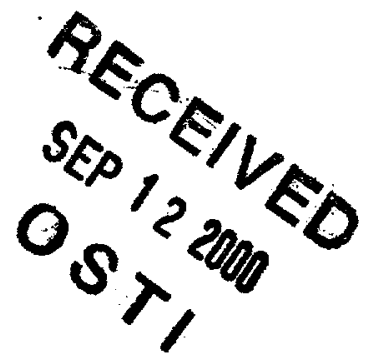

July 2000

\begin{abstract}
The submitted manuscript has been created by
the University of Chicago as Operator of

Argonne National Laboratory ("Argonne") under

Contract No. W-31-109-ENG-38 with the U.S.

Department of Energy. The U.S. Government

retains for itself, and others acting on its behalf,

a paid-up, nonexclusive, irrevocable worldwide

license in said article to reproduce, prepare

derivative works, distribute copies to the public,

and perform publicly and display publicly, by

or on behalf of the Government.
\end{abstract}

Conference paper that was presented " 8 th International Conference on the structure of non-

crystalline solids" University of Wales, England, will appear in Journal of Non-crystalline Solids.

\footnotetext{
${ }^{*}$ This work is supported by the U.S. Department of Energy, Basic Energy Sciences, Office of Science, under contract \#W-31-109-ENG-38.
} 


\section{DISCLAIMER}

This report was prepared as an account of work sponsored by an agency of the United States Government. Neither the United States Government nor any agency thereof, nor any of their employees, make any warranty, express or implied, or assumes any legal liability or responsibility for the accuracy, completeness, or usefulness of any information, apparatus, product, or process disclosed, or represents that its use would not infringe privately owned rights. Reference herein to any specific commercial product, process, or service by trade name, trademark, manufacturer, or otherwise does not necessarily constitute or imply its endorsement, recommendation, or favoring by the United States Government or any agency thereof. The views and opinions of authors expressed herein do not necessarily state or reflect those of the United States Government or any agency thereof. 


\section{DISCLAIMER}

\section{Portions of this document may be illegible in electronic image products. Images are produced from the best available original document.}




\title{
High-resolution atomic distribution functions of disordered materials by high-energy $x$-ray diffraction
}

\author{
V. Petkov" ${ }^{2 *}$, S. J.L. Billinge ${ }^{a}$, S. D. Shastri ${ }^{b}$ and B. Himmel ${ }^{c}$ \\ ${ }^{a}$ Department of Physics and Astronomy and Center for Fundamental Materials \\ Research, Michigan State University, East Lansing, MI-48823, USA \\ ${ }^{b}$ Advanced Photon Source, Argonne National Laboratory, Argonne, IL-60439, USA \\ ${ }^{c} F B$ Elektrotechnik and Informationtechnik, Universit ät Rostock, Rostock, GERMANY
}

\begin{abstract}
The atomic-scale structure of materials with different degrees of structural disorder has been investigated by high-energy $(\mathrm{E} \geq 60 \mathrm{keV}) \mathrm{x}$-ray diffraction. Good quality structure functions extended to wave vectors of magnitude $Q \geq 40 \AA^{-1}$ have been obtained even in case of materials composed of weakly scattering, light atomic species. The corresponding high-resolution atomic distribution functions allowed fine structural features differing in as little as $0.14 \AA$ to be resolved. In particular, the distinct $G a-A s(\sim 2.43 \AA)$ and $I n-A s(2.61 \AA)$ bonds in $I n-G a-A s$ alloys have been differentiated. The atomic scale structure of "restacked" $W S_{2}$ has been determined and the presence of three distinct $W-W$ distances occurring at $2.77 \AA, 3.27 \AA$ and $3.85 \AA$ established. Finally, Si-O $(\sim 1.61 \AA), A l-O(\sim 1.75 \AA)$ and $\mathrm{Ca}-O(\sim 2.34 \AA)$ bonds in calcium aluminosilicate glasses have been differentiated and the characteristics of the respective coordination polyhedra revealed.
\end{abstract}

"Corresponding author: Michigan State University, Department of Physics and Astronomy, East Lansing, MI 48823. Tel.: +1-517353 5288; fax: +1-517353 4500;

e-mail: petkov@pa.msu.edu 


\section{Introduction}

Knowledge of the atomic-scale structure is a prerequisite to understanding the physical properties and technological characteristics of materials. Also, many technologically important materials contain significant disorder on an atomic scale. Often this disorder has a direct effect on the properties which make the material technologically and/or scientifically important. It is clearly advantageous to characterize the structure of disordered materials with high resolution. A fruitful experimental approach is the combination of the high-energy $x$-ray diffraction and the atomic Pair Distribution Function (PDF) technique.

The atomic PDF, $G(r)=4 \pi\left[\rho(r)-\rho_{o}\right]$, is the sine Fourier transform of the experimentally observable total structure function, $S(Q)$, i.e.

$$
G(r)=(2 / \pi) \int_{Q=0}^{Q_{\operatorname{man}}} Q[S(Q)-1] \sin (Q r) d Q,
$$

where and $r$ is the radial distance, $Q$ the magnitude of the wave vector and $\rho(r)$ and $\rho_{o}$ the local and average atomic number densities, respectively. The structure function is related to the elastic part of the total diffracted intensity, $I^{\text {el. }}(Q)$, as follows:

$$
S(Q)=1+\left[I^{e l}(Q)-\sum c_{i}\left|f_{i}(Q)\right|^{2}\right] /\left|\sum c_{i} f_{i}(Q)\right|^{2},
$$

where $I^{\text {coh. }}(Q)$ is the coherent scattering intensity per atom in electron units and $c_{\mathrm{i}}$ and $f_{i}$ are the atomic concentration and scattering factor respectively for the atomic species of type $i$ [1]. Two important advantages of the atomic PDFs are to be noted: First, the PDF is obtained with no assumption of periodicity and, therefore, glassy materials as well as polycrystals exhibiting different degrees of structural disorder can be studied applying the same approach. Second, by using highenergy x-rays high values of $Q$ can be assessed. This results in PDFs with improved resolution allowing quite fine structural features to be revealed. The advantages are well demonstrated by the results presented below. 


\section{Results}

\subsection{Local structural disorder in In-Ga-As semiconductor alloys}

$I n_{x} G a_{1-x} A s$ alloys are technologically important because they allow the semiconductor band gap to be varied continuously between the band gap values of the end members by varying the composition, $x$. XAFS experiments [2] have shown that $I n-A s$ and $G a-A s$ bond lengths do not take average values but remain close to their natural lengths $L_{G a-A s}=2.43 \AA$ and $L_{l n-A s}=2.61 \AA$ in the alloy. This finding shows that the underlying zinc-blende lattice must distort significantly to accommodate the two distinct bonds length present in the alloys. Since the structural distortion affects the alloy's properties it warrants a detailed study. The study was carried out at the A2 beam line, The Cornell High Energy Synchrotron Source (CHESS), using $x$-rays of energy $60 \mathrm{keV}$ [3]. Two of the extended structure functions obtained are shown in Fig. 1. Significant Bragg scattering (well-defined peaks) is present 1 up to $Q \sim 35 \AA^{-1}$ in the end member GaAs. The material has a perfect crystalline order. The Bragg peaks disappear at much lower $Q$ values in the $I n_{0.5} G a_{0.5} A s$ sample. At high-Q values, only oscillating diffuse scattering is seen. The alloy obviously exhibits considerable structural disorder. The two different bond lengths that cause it are well seen as a split first peak in the corresponding $\mathrm{G}(\mathrm{r})$ also shown in Fig. 1. To quantify the distortions in the alloy, simple models based on the 8atom unit cell of the zinc-blende lattice were fit to the experimental PDFs [4]. The PDF for GaAs is fit well with a model based on the perfect zinc-blende structure as can be seen in Fig. 1. However, definitive static displacement of the atoms from their sites in the ideal zinc-blende lattice were necessary to be introduced in the model structure to fit the PDF for $\operatorname{In}_{0.5} G a_{0.5} A s$. The refined discrete displacements on $A s$ and metal $(I n ; G a)$ sites amounted to $0.133(5) \AA$ and $0.033(8) \AA$, respectively. Fragments of the refined structure models are illustrated in Fig. 2. The model result show that most of the structural distortion occurs by arsenic moving off its site, but displacement of metal atoms are also important $[3,4]$. 


\subsection{Structure of restacked $W_{2}$}

Layered $W S_{2}$ shows fascinating chemical properties. It can be easily exfoliated to form a suspension of single $\left(W_{2}\right)$ layers and then restacked, with foreign species included if desired [5]. Xray diffraction experiments were carried out to determine the structure of restacked $W S_{2}$ since it would help in understanding the material's guest-host and catalytic properties. The experiments were carried out at the A2 beam line, CHESS, using $x$-rays of energy $60 \mathrm{keV}$. A complementary experiment on pristine $W_{2}$ was also carried out with the use of laboratory source of $x$-rays with $\mathrm{Ag}$ anode. The experimental structure functions and atomic PDFs obtained are shown in Fig. 3. The structure function of pristine $W S_{2}$ consists of well-defined Bragg peaks - a characteristic typically seen in well-crystalline materials. The corresponding atomic PDF is perfectly fit with the wellknown structure of hexagonal $W S_{2}[6]$. The structure function of restacked $W S_{2}$ contains only a few sharp peaks and a pronounced diffuse component - a characteristic of a significantly disordered , material. Such a featureless experimental structure function is practically impossible to be tackled by ordinary techniques for structure determination including the Rietveld technique. The corresponding PDF, however, is rich in structure-related features and lends itself to structure determination. Several structure models were tested and it was found that the structure of restacked $W S_{2}$ is best described on the basis of a monoclinic unit cell being a distorted derivative of the 8-atom unit cell of orthorhombic $W T e_{2}[6]$. The quality of this structure model is well demonstrated in fig. 3. A fragment of it is given in fig. 4. Thus, although restacked $W S_{2}$ is quite disordered it still possesses three-dimensional order, i.e. it is not simply a turbostratic pile-up of $\left(W S_{2}\right)$ monolayers. The structures of pristine and restacked $W S_{2}$ differ most in the spatial arrangement of metal atoms. Tungsten atoms occupy a single site and are separated by the same distance of $3.18 \AA$ in pristine $W S_{2}$. They occupy two distinct sites in restacked $W S_{2}$. As a result three distinct $W-W$ atomic-pair

distances occur at $2.77 \AA, 3.85 \AA$ and $3.27 \AA$, respectively. These distinct $W$-W pairs are what make 
the low-r region of the PDF for restacked $W S_{2}$ look so different from the one of the PDF for pristine $W S_{2}[7]$.

\subsection{Polyhedral units and network connectivity in calcium aluminosilicate glasses}

Silicate glasses in general, and calcium aluminosilicates glasses in particular, are of great industrial and technological importance. It is well known that silica glass is a continuous network of corner-shared $\mathrm{SiO}_{4}$ tetrahedra. When $\mathrm{Sl}^{4+}$ is replaced by $\mathrm{Al}^{+3}$ and $\mathrm{Ca}^{+2}$ is introduced to balance the charge the glassy structure changes. The change involves a replacement of $\mathrm{Si}-\mathrm{O}$ polyhedra by $\mathrm{Al}-\mathrm{O}$ ones and an emerging of so-called non-bridging oxygens (NBO) linking a $\mathrm{Si}-\mathrm{O}$ or $\mathrm{Al}-\mathrm{O}$ unit with a $\mathrm{Ca}$-O one [8]. Important issues about the modifier $(\mathrm{Ca})$ ion coordination and the nature of $N B O$ 's that break the network are, however, not so well established. To fill this gap a study on the atomic ordering in a series of $\mathrm{Ca}_{x 2} \mathrm{Al}_{x} \mathrm{Si}_{l_{-x}} \mathrm{O}_{2}$ glasses $(\mathrm{x}=0.0 .25,0.5,0.67)$ was undertaken. The experiments were carried out at the 1-ID beam line, The Advanced Photon Source, Argonne, with the use of $x$ rays of energy $80.6 \mathrm{keV}$. The structure functions obtained are shown in fig. 5 . They exhibit prominent oscillations up to the maximum $Q$ value of $40 \AA^{-1}$ reached. The oscillations may only come from the presence of well-defined polyhedral units building the glasses. These polyhedra are seen as well defined peaks in the low-r region of the experimental PDFs shown in fig. 6 . By fitting the peaks with gaussians the corresponding coordination numbers and radii were estimated. It is found that both $S i$ and $A l$ are coordinated by four oxygens and thus participate in a continuous tetrahedral network when $A / / C a$ content is low $(x=0,0.25)$. By $\mathrm{x}=0.5$ the network begins to break. It is indicated by the slight drop in the average coordination number of $S i$ from $4.0(1)$ to $3.85(1)$. When more than half of $S i$ is replaced by $A l(x=0.67)$ the number of NBO's in the first coordination sphere of $\mathrm{Si}$ increases making the average $\mathrm{Si}-\mathrm{O}$ coordination number as low as 3.2(1). Al-O average coordination number is found to be close to 4.0 (1) for all values of $x$. Thus Al-O tetrahedra are 
always free of NBO's and fully integrated in the network. $\mathrm{Ca}-\mathrm{O}$ coordination is found to be well defined and close to $5.0(1)$ for all values of $\mathrm{x}$. The result implies that not only Si/Al-O tetrahedra but $\mathrm{Ca}$ polyhedra, too, play a role in the formation of the glassy structure [10].

\section{Conclusion}

Good quality, high-resolution atomic PDFs can be obtained by high-energy $x$-ray diffraction even in case of materials composed of weakly scattering, light atomic species. Thus the structure of materials exhibiting different degrees of structural disorder can be studied with improved resolution. These could be crystalline materials such as In-Ga-As alloys where a small deviation from the perfect order is present, heavily disordered but still three-dimensionally periodic materials such as restacked $W S_{2}$ and completely disordered materials such as calcium aluminosilicate glasses. High-resolution PDFs are expected to become more frequently employed in structure studies with the number of synchrotron sources rapidly increasing worldwide.

\section{Acknowledgements}

The semiconductor alloy work was supported by DOE grant DE FG02 97ER45651 and the WS 2 and glasses work by NSF grant CHE 99-03706. CHESS is operated by NSF through grant DMR9713242. The Advanced Photon Source is operated by DOE under contract W-31-109-Eng-38. Thanks

are due to S. Kycia, I-K. Jeong, F. Muhiuddin-Jacobs, Th. Proffen, J. Heising and M. Kanatzidis for the fruitful cooperation.

\section{References}

1. B.E. Warren, X-ray diffraction (Dover, New York, 1990).

2. J.C. Mikkelson and J.B. Boyce, Phys. Rev. Lett. 49 (1982) 1412. 
3. V. Petkov, I-K. Jeong, J.S. Chung, M.F. Thorpe, S. Kycia and S.J.L. Billinge, Phys. Rev. Lett. 83 (1999) 4089.

4. V. Petkov, I-K. Jeong, F. Muhiuddin-Jacobs, Th. Proffen, J.S.L. Billinge and W. Dmowski, J. Appl. Phys. 88 (2000)

5. W.M.R. Divigalpitiya, R.F. Frindt and S.R. Morrison, Science 246 (1989) 369.

6. R.W.G. Wyckoff, in Crystal Structures (John Wiley \& Sons, New York 1964).

7. V. Petkov, S.J.L. Billinge, J. Heising and M. Kanatzidis, J. Am. Chem. Soc. (2000), submitted.

8. B.O. Mysen, in Structure and properties of silicate melts (Elsevier, Amsterdam, 1988).

9. V. Petkov and R. Danev, J. Appl. Cryst. 31 (1998) 609.

10. V. Petkov, S.J.L. Billinge, S.D. Shastri and B. Himmel, Phys. Rev. Lett. (2000), submitted. 


\section{Figure captions}

Figure 1. Reduced structure functions $Q[S(Q)-1]$ (upper part) and the corresponding high-resolution atomic PDFs (symbols; lower part) for GaAs and $I n_{0.5} G a_{0 . s A s}$. Model PDFs (full line; lower part) are based on perfect (for $G a A s$ ) and distorted (for $I n_{0.5} G a_{0.5 A s}$ ) zinc-blende lattices.

Figure 2. Schematic representation of the local structural distortions in $\ln _{0.5} G a_{0.5} A s$. Fragments of the perfect lattice (thin line) can be compared with the distorted one (thick line). The view is a projection along the $b$ axis of the unit cell. The large circles are As sites and the small circles the metal $(\mathrm{In} ; \mathrm{Ga})$ sites.

Figure 3. Reduced structure functions $Q[S(Q)-1]$ (upper part) and the corresponding atomic PDFs (symbols; lower part) for pristine and restacked $W S_{2}$. Model PDFs (full line; lower part) are based on hexagonal (for pristine $W S_{2}$ ) and monoclinic (for restacked $W S_{2}$ ) structures as described in the text.

Figure 4. Fragment of the structure of restacked $W S_{2}$ with the monoclinic unit cell shown. The big circles are $W$ atoms and the small ones $-S$ atoms.

Figure 5. Reduced structure functions $Q[S(Q)-1]$ for calcium aluminosilicate glasses (symbols) together with the optimum smooth line placed with the help of the Maximum Entropy Method [9].

Figure 6. Gaussian fit to the first peaks in the atomic PDFs, $g(r)=\rho(r) / \rho_{o}$, for $\mathrm{Ca}_{x / 2} \mathrm{Al}_{\mathrm{x}} \mathrm{Si}_{1-\mathrm{x}} \mathrm{O}_{2}$ glasses $(x=0 ., 0.25,0.5,0.67)$. Experimental data: symbols; fitted data: full line; individual gaussians: broken line; residual difference: full line (bottom). Peaks are labeled with the corresponding atomic pairs. 

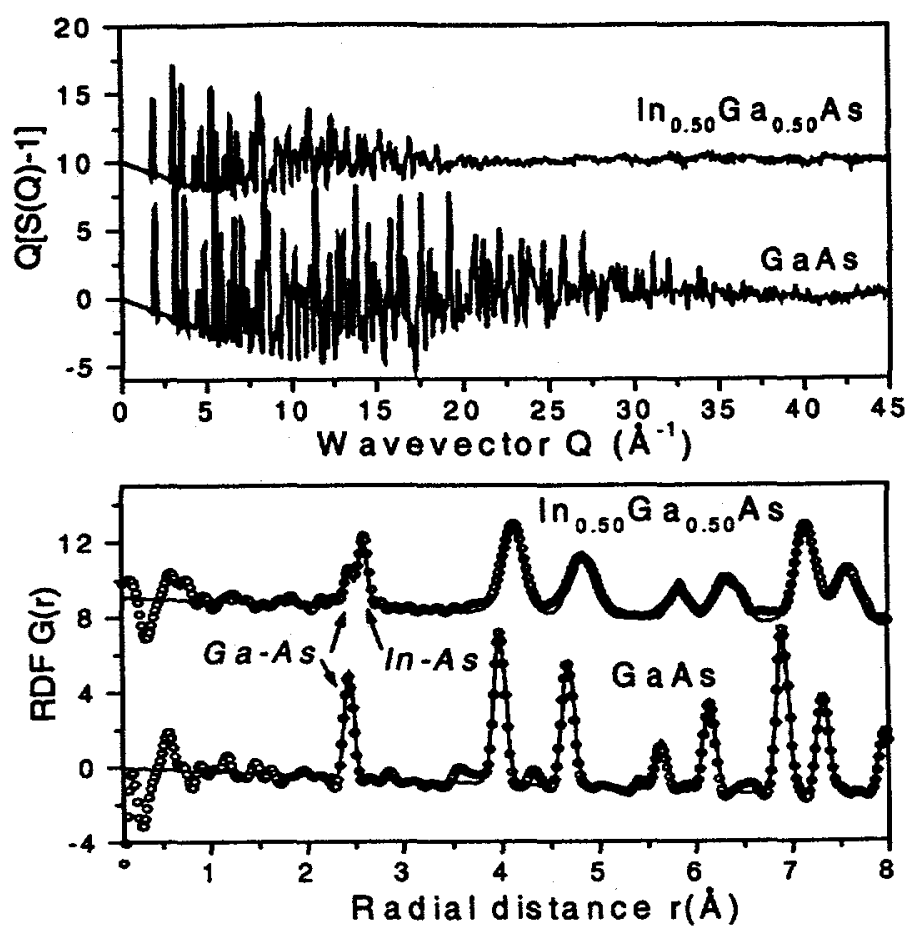

Figure 1.

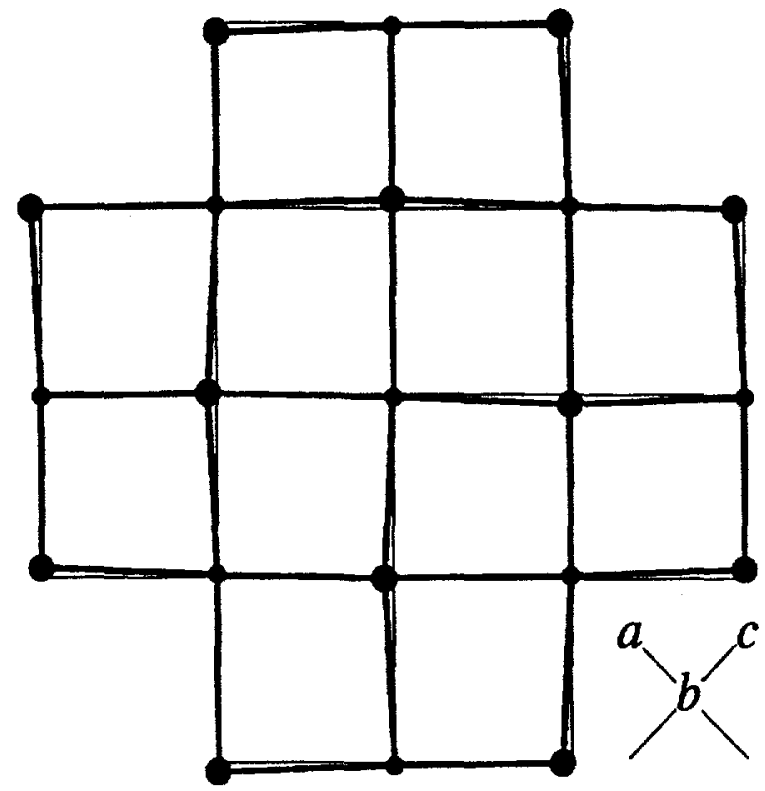

Figure 2 

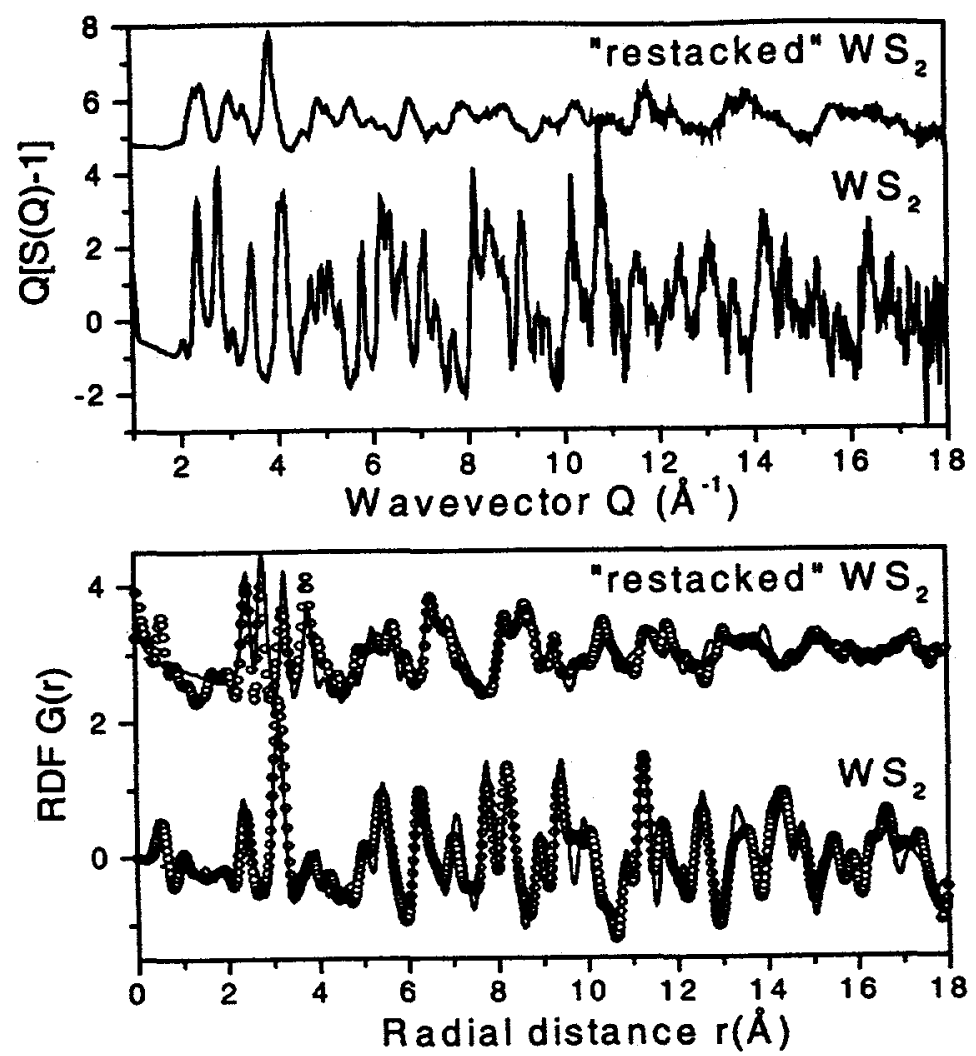

Figure 3

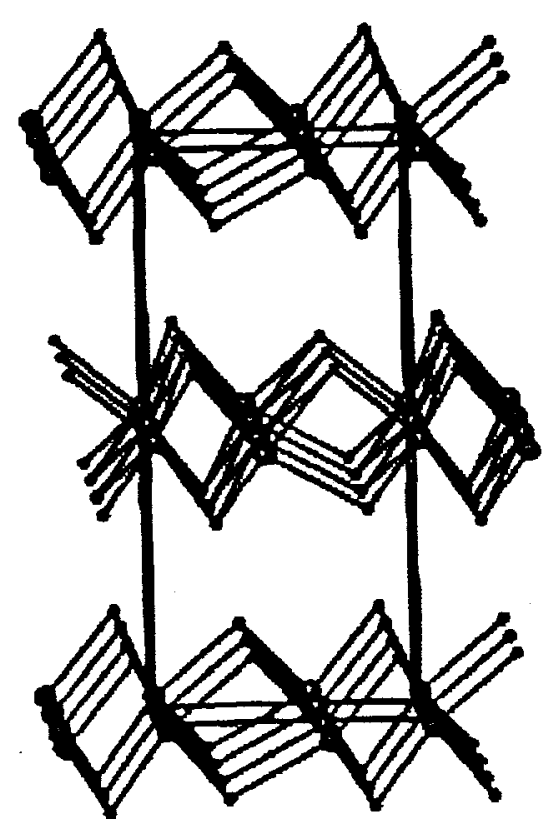

Figure 4 


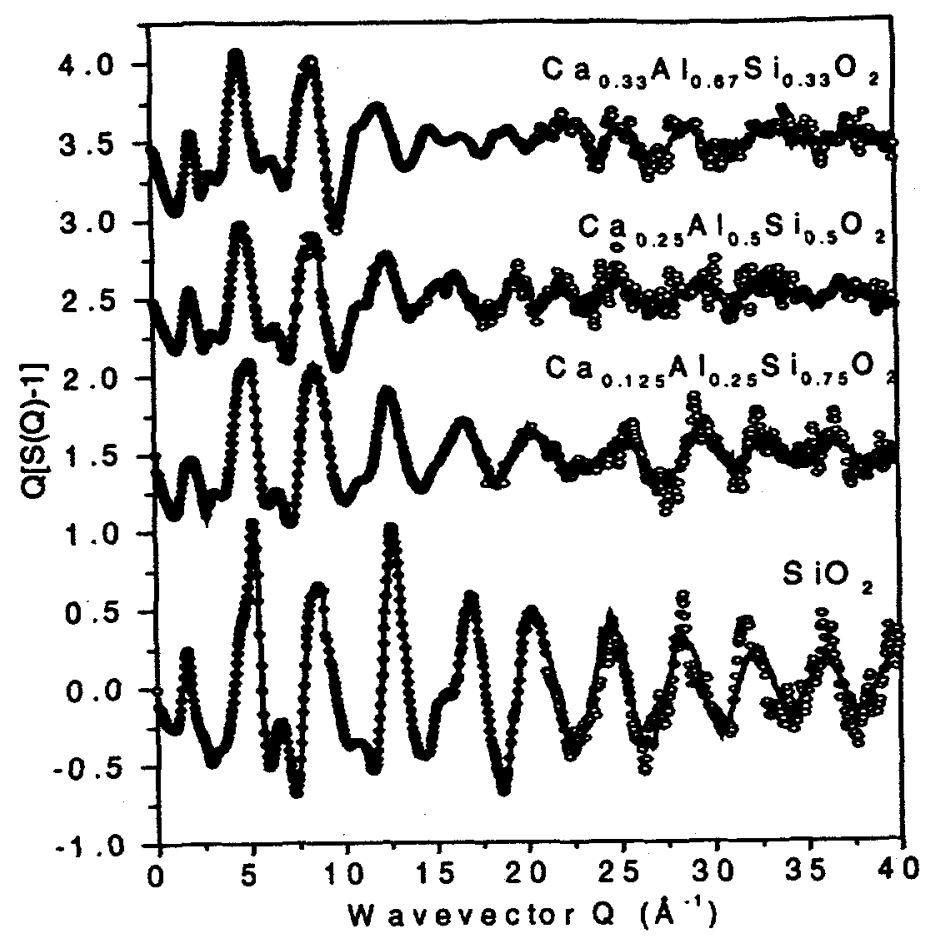

Figure 5

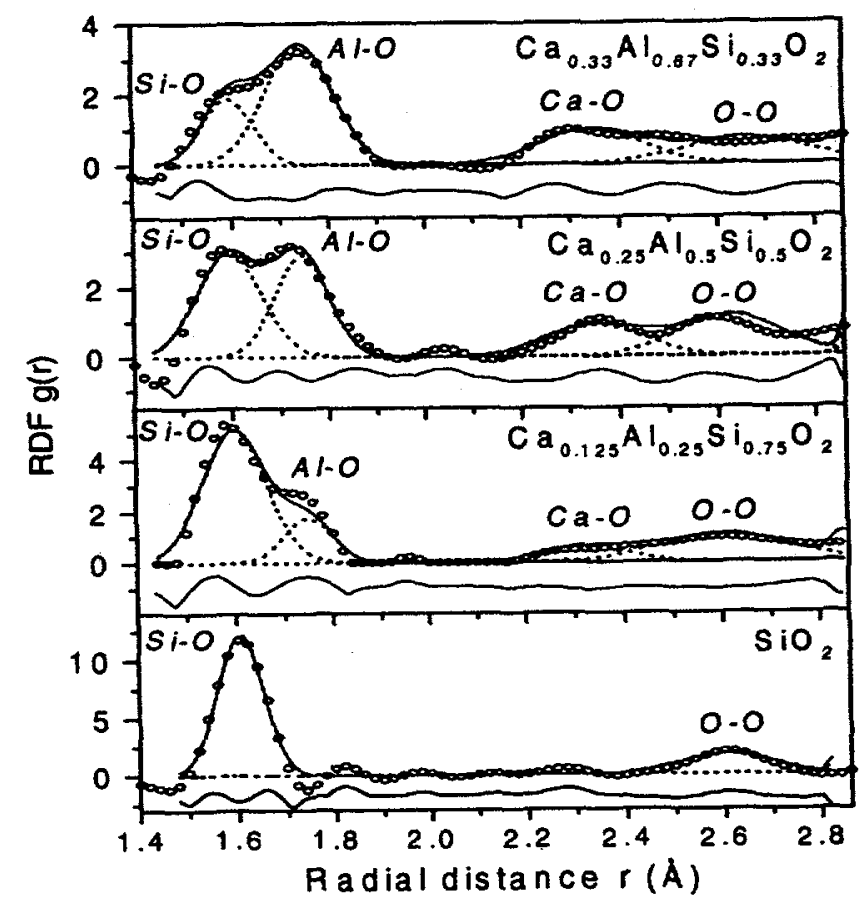

Figure 6 